\title{
Economía, economistas y expectativas
}

Título: Economics, Economists, and Expectation. Microfoundations to macroapplications Autores: Warren Young, Robert Lesson and William Darity Jnr.

Edición: Routledge Studies in the History of Economics, London \& New York, 2004, 160 pp. ISBN. 978-0415085151

Elena MÁRQUEZ DE LA CRUZ

Universidad Complutense de Madrid

El papel crucial que las expectativas de los agentes económicos juegan en la modelización del comportamiento de éstos genera, hoy en día, poca controversia. La denominada revolución de las expectativas racionales supuso un cambio de enfoque en el análisis de los procesos de decisión de los agentes económicos, así como, y como resultado de lo anterior, en el de la efectividad de las medidas de política económica, dando paso a una nueva etapa en el desarrollo de los modelos macroeconómicos. Sin embargo, uno de los aspectos más problemáticos relacionado con las expectativas es su tratamiento por parte de los historiadores del pensamiento económico. Así, a lo largo de las 160 páginas de este libro, los autores muestran las múltiples lagunas y omisiones que se pueden encontrar en trabajos anteriores acerca de cómo la idea de las expectativas racionales se desarrolló y consolidó como eje esencial de nuestra disciplina. Tanto es así, que los autores afirman que la denominada revolución de las expectativas racionales no fue tal, toda vez que sus fundamentos teóricos ya se había expuesto y empleado mucho tiempo antes de que tal revolución tuviera lugar.

En los primeros capítulos del libro, el lector descubrirá la aparición de ideas relacionadas con las expectativas en los trabajos de Hayek (primero en diferenciar entre los aspectos individuales y agregados de las expectativas), Hicks (para quien el desequilibrio no es más que la frustración de las expectativas) o Kaldor (que concluye que la inestabilidad es el resultado de unas expectativas erróneas). Por su parte, Morgenstern trató ampliamente el tema de la racionalidad de los agentes, estableciendo lo que, tiempo después, vendría a ser la versión fuerte de las expectativas racionales. Y sin ánimo de exhaustividad, las ideas de Pigou, la diferente posición de Keynes en A Treatise y The General Theory en lo que al conocimiento sobre las variables futuras se refiere, la propuesta de una relación entre información "completa" y "perfecta" y comportamiento racional de Von Neumann y Morgenstern en 1944, y la aparición implícita de la idea de las expectativas racionales en la teoría de juegos desarrollada por ambos, y continuada en los trabajos de 1950 de Marschak y Nash (que llevaría décadas después a diferenciar entre expectativas racionales en el sentido de Muth y expectativas racionales en el sentido de Nash), son algunos de los aspectos que los autores tratan con detalle. Pero, sin duda, en el desarrollo de las expectativas racionales, el trabajo de Hart sobre las previsiones y la empresa merece una especial consideración a criterio de los autores. Y es que en 1949, Hart ya plantea la misma premisa en la que se basarían Lucas, Sargent y Wallace dos décadas después: lo inverosímil del supuesto implícito de invariabilidad del modelo de estimación cuando el entorno económico cambia.

Entre 1949, cuando el proyecto de Illinois se discutió por primera vez en la AEA Meeting (por, entre otros, Hart, Modigliani y Friedman), y 1959, cuando Muth y Mills presentan sus respectivos trabajos acerca de las expectativas racionales en la Econometric Society, se desarrollaron diversos proyectos sobre expectativas tanto en Estados Unidos como en el Reino Unido (a éstos y otros muchos se dedica el capítulo 3). Los proyectos "formales" se desarrollaron en diversas universidades con el objetivo específico de estudiar el papel de las expectativas; a los proyectos de Illinois y Carnegie Tech se dedica un tratamiento destacado. Los diferentes proyectos de investigación desarrollados tenían un claro enfoque empírico, si bien todos ellos buscaban la formulación del soporte teórico de la idea de expectativas. Uno de los economistas destacados en ambos proyectos fue Modigliani (que se trasladó de Illinois a Carnegie Tech) quien, en 1954, anticipa a Lucas al señalar que: "[...] el argumento de este trabajo sugiere que la 
reacción de los agentes puede generar dificultades para la elaboración y ejecución de las políticas".

Rescatar la contribución de Mills a la formulación y desarrollo de la idea de las expectativas racionales es otra de las tareas que se proponen los autores (y a la que dedican buena parte del capítulo 4). Baste la siguiente afirmación para entender hasta qué punto Mills fue, en su opinión, crucial:

[...] Quizá, si se hubiese prestado más atención al trabajo que Mills desarrollaba durante el período 1954/55-1957/59, la así denominada "Revolución de las Expectativas Racionales" habría tenido lugar más de una década antes de cuando en realidad se produjo!

Aunque el trabajo de Muth del año 1961 se considera el punto de partida de la revolución de las expectativas racionales, el capítulo 5 nos muestra que, ya en 1932, Tinberger fue el primero, y durante casi 30 años el único, que relacionó la teoría de la economía dinámica con las expectativas, la incertidumbre y la teoría de la probabilidad. De hecho, el modelo de Tinberger del año 1932 puede considerarse predecesor del de Muth de 1961, si bien los autores reconocen la existencia de diferencias entre ambos: mientras que Muth centró su atención en procesos autocorrelacionados, Tinberger sólo analizó el caso de perturbaciones serialmente independientes. Pero lo esencial para ellos es que el trabajo de Muth no puede entenderse sin, de nuevo, hacer referencia a Mills; no en vano, el propio Muth tuvo que adoptar el enfoque de Mills como solución en la modelización de las expectativas a nivel microeconómico, debido a los problemas relacionados con los errores de medida cuando su propio enfoque era empleado. Todo lo cual nos lleva de nuevo a que, la revolución, si es que existió, tuvo lugar mucho antes de lo que se considera.

Patinkin es el protagonista del capítulo 6, en el que se lleva a cabo un detallado estudio de las distintas ediciones de Money, Interest and Prices con el objetivo de explicar qué razones pudieron llevar a Patinkin a optar por un esquema de expectativas adaptativas a pesar de que las expectativas racionales estaban presentes y eran de clara aplicación en el contexto de su modelo. La razón es explicada por el propio Patinkin en la edición resumida de finales de los años 80. La idea esencial esgrimida es que las expectativas racionales son incompatibles con el enfoque de desequilibrio que él pretendía desarrollar; sin embargo, las expectativas adaptativas permiten la estabilidad del sistema macroeconómico en desequilibrio que propone, dado que hacen posible la existencia de errores sistemáticos a un nivel microeconómico.

El libro se cierra con el capítulo dedicado a la contrarrevolución monetarista, con un minucioso análisis de las aportaciones de Phillips, Friedman y, como no puede ser de otro modo, con la definitiva contribución de Lucas. Es ésta la parte del libro más conocida por los economistas $\mathrm{y}$, por tanto, menos sorprendente, toda vez que sus contenidos forman parte de los manuales de macroeconomía al uso.

En definitiva, un libro de lectura esencial para todo el que desee lograr una conocimiento profundo sobre uno de los temas clave en economía, no sólo, aunque sí especialmente, desde la perspectiva de la historia del pensamiento económico. El lector no encontrará una revisión de la literatura al uso acerca de las aplicaciones de las expectativas racionales al análisis económico, sino la demostración de que la revolución de las expectativas racionales, si es que existió, tuvo lugar mucho antes de lo que se nos ha hecho creer. 